Pacific

Journal of

Mathematics

\title{
TWO INFINITE VERSIONS OF THE NONLINEAR DVORETZKY THEOREM
}

KeI FunANO

Volume 259 No. 1

September 2012 


\title{
TWO INFINITE VERSIONS OF THE NONLINEAR DVORETZKY THEOREM
}

\author{
KEI FUNANO
}

\begin{abstract}
We make two additions to recent results of Mendel and Naor on nonlinear versions of Dvoretzky's theorem. We consider the cases of metric spaces with infinite Hausdorff dimension and countably infinite metric spaces.
\end{abstract}

\section{Introduction and the statement of the results}

We say that a metric space $X$ is embedded with distortion $D \geq 1$ in a metric space $Y$ if there exist a map $f: X \rightarrow Y$ and a constant $r>0$ such that

$$
r d_{X}(x, y) \leq d_{Y}(f(x), f(y)) \leq \operatorname{Dr} d_{X}(x, y) \text { for all } x, y \in X .
$$

Such a map $f$ is called a $D$-embedding.

Dvoretzky's theorem [1961] states that for every $\varepsilon>0$, every $n$-dimensional normed space contains a $k(n, \varepsilon)$-dimensional subspace that embeds into a Hilbert space with distortion $1+\varepsilon$. This theorem was conjectured by Grothendieck [1953]. See [Milman 1971; 1992; Milman and Schechtman 1999; Schechtman 2006; 2011] for the estimates of $k(n, \varepsilon)$ and the further developments related to this theorem.

Bourgain, Figiel, and Milman proved a natural nonlinear variant of Dvoretzky's theorem:

Theorem 1.1 [Bourgain et al. 1986]. There exists two universal constants $c_{1}, c_{2}>0$ such that for every $\varepsilon>0$, every finite metric space X contains a subset $S$ that embeds into a Hilbert space with distortion $1+\varepsilon$ and

$$
|S| \geq \frac{c_{1} \varepsilon}{\log \left(c_{2} / \varepsilon\right)} \log |X|
$$

See [Bartal et al. 2005; Mendel and Naor 2007; Naor and Tao 2012] for further discussion. It is natural to try to get some versions of the above theorem in the case where $|X|=\infty$. In this paper we prove the following.

This work was partially supported by Grant-in-Aid for Research Activity (startup), grant number 23840020.

MSC2010: 53C23.

Keywords: Dvoretzky's theorem, ultrametric space. 
Theorem 1.2. For every $\varepsilon>0$, every countable infinite metric space $X$ has an infinite subset which embeds into an ultrametric space with distortion $1+\varepsilon$.

Recall that a metric space $(U, \rho)$ is called an ultrametric space if for every $x, y, z \in X$ we have $\rho(x, y) \leq \max \{\rho(x, z), \rho(z, y)\}$. Since every separable ultrametric space isometrically embeds into a Hilbert space [Vestfrid and Timan 1979], we verify that Theorem 1.1 holds in the case where $|X|=\infty$.

Recently Mendel and Naor [2012] proved another variant of Dvoretzky's theorem, answering a question by T. Tao. For a metric space $X$ we denote its Hausdorff dimension by $\operatorname{dim}_{H} X$. A subset of a complete separable metric space is called an analytic set if it is an image of a complete separable metric space under a continuous map. Note that analytic sets are not necessarily complete. For example, any Borel subset of a complete separable metric space is an analytic set (refer to [Kechris 1995] for analytic sets).

Theorem 1.3 (compare [Mendel and Naor 2012, Theorem 1.7]). There exists a universal constant $c \in(0, \infty)$ such that for every $\varepsilon \in(0, \infty)$, every analytic set $X$ whose Hausdorff dimension is finite has a closed subset $S \subseteq X$ that embeds with distortion $2+\varepsilon$ in an ultrametric space, and

$$
\operatorname{dim}_{H} S \geq \frac{c \varepsilon}{\log (1 / \varepsilon)} \operatorname{dim}_{H} X .
$$

Mendel and Naor [2012] stated this theorem only for compact metric spaces. As remarked in their introduction, the theorem is valid for more general metric spaces. For example, the theorem holds for every analytic set $X$, since the problem can be reduced to the case of a compact subset of $X$ with the same Hausdorff dimension (see [Carleson 1967; Howroyd 1995, Corollary 7]).

In the following theorem we consider the case where $\operatorname{dim}_{H} X=\infty$.

Theorem 1.4. For every $\varepsilon \in(0, \infty)$, every analytic set $X$ whose Hausdorff dimension is infinite has a closed subset $S$ that can be embedded into an ultrametric space with distortion $2+\varepsilon$ and has infinite Hausdorff dimension.

It follows from the proof of Theorem 1.3 in [Mendel and Naor 2012] that if $\operatorname{dim}_{H} X=\infty$, then $X$ contains an arbitrary large-dimensional closed subset that embeds into an ultrametric space. Combining Theorem 1.3 with Theorem 1.4 we find that a nonlinear Dvoretzky theorem holds for all analytic sets.

The following theorem asserts that the distortion in Theorems 1.3 and 1.4 cannot be strictly less than two.

Theorem 1.5 [Mendel and Naor 2012, Theorem 1.8]. For every $\alpha>0$ there exists a compact metric space $(X, d)$ of Hausdorff dimension $\alpha$, such that if $S \subseteq X$ embeds into a Hilbert space with distortion strictly smaller than 2 then $\operatorname{dim}_{H} S=0$. 
Theorem 1.5 immediately implies that the same result holds in the case $\alpha=\infty$.

It is known that $\ell_{2}$ does not embed into $\ell_{p}$ with finite distortion for any $p$ in $[1, \infty) \backslash\{2\}$ [Albiac and Kalton 2006, Corollary 2.1.6]. In particular, an infinitedimensional analogue of Dvoretzky's theorem is no longer true in the linear setting. In contrast to this fact, Theorem 1.4 asserts that an infinite-dimensional Dvoretzky theorem holds in the nonlinear setting.

\section{Proof}

Lemma 2.1. Let $X$ be a separable metric space such that $\operatorname{dim}_{H} X=\infty$. Then there exists a sequence $\left\{K_{i}\right\}_{i=1}^{\infty}$ of mutually disjoint closed subsets of $X$ such that

$$
\lim _{i \rightarrow \infty} \operatorname{diam} K_{i}=0 \quad \text { and } \quad \lim _{i \rightarrow \infty} \operatorname{dim}_{H} K_{i}=\infty .
$$

Proof. For every $x \in X$ we take a closed neighborhood $K_{x}$ of $x$ with diam $K_{x} \leq 1$. Since $X$ is separable, applying the Lindelöf covering theorem we get a countable subset $F \subseteq X$ such that $X=\bigcup_{x \in F} K_{x}$. Since

$$
\operatorname{dim}_{H}\left(\bigcup_{x \in F} K_{x}\right)=\sup _{x \in F} \operatorname{dim}_{H} K_{x}
$$

there exists $x_{1} \in F$ such that $\operatorname{dim}_{H} K_{x_{1}}=\infty$ or there exists a sequence $\left\{y_{i}\right\}_{i=1}^{\infty} \subseteq F$ such that $\left\{\operatorname{dim}_{H} K_{y_{i}}\right\}_{i=1}^{\infty}$ is strictly increasing and $\lim _{i \rightarrow \infty} \operatorname{dim}_{H} K_{y_{i}}=\infty$.

We first consider the latter case. We put $K_{1}:=K_{y_{1}}$. By the monotonicity of $\operatorname{dim}_{H} K_{y_{i}}$ we have $\operatorname{dim}_{H}\left(K_{y_{i}} \backslash \bigcup_{j=1}^{i-1} K_{y_{j}}\right)=\operatorname{dim}_{H} K_{y_{i}}$ for $i \geq 2$. Covering $K_{y_{i}} \backslash \bigcup_{j=1}^{i-1} K_{y_{j}}$ by countably many closed subsets of diameter $\leq 1 / i$, we thus find a closed subset $K_{i} \subseteq K_{y_{i}} \backslash \bigcup_{j=1}^{i-1} K_{y_{j}}$ such that $\operatorname{dim}_{H} K_{i}=\operatorname{dim}_{H} K_{y_{i}}$ and diam $K_{i} \leq 1 / i$. This $\left\{K_{i}\right\}_{i=1}^{\infty}$ is the desired sequence.

We now consider the former case. Covering $K_{x_{1}}$ by countably many closed subsets $\left\{K_{y}^{1}\right\}_{y \in F_{1}}$ so that diam $K_{y}^{1} \leq 2^{-1} \operatorname{diam} K_{x_{1}}$, we have two cases: There exists $x_{2} \in F_{1}$ such that $\operatorname{dim}_{H} K_{x_{2}}^{1}=\infty$ or there exists a sequence $\left\{y_{i}\right\}_{i=1}^{\infty} \subseteq F_{1}$ such that $\left\{\operatorname{dim}_{H} K_{y_{i}}^{1}\right\}_{i=1}^{\infty}$ is strictly increasing and $\lim _{i \rightarrow \infty} \operatorname{dim}_{H} K_{y_{i}}^{1}=\infty$. Since we have already proved the lemma in the latter case, we consider the former case. Continuing this process we may assume there is a chain $K_{x_{2}}^{1} \supseteq K_{x_{3}}^{2} \supseteq K_{x_{4}}^{3} \supseteq \cdots$ of closed subsets of $X$ such that

$$
\operatorname{dim}_{H} K_{x_{i}}^{i-1}=\infty \quad \text { and } \quad \operatorname{diam} K_{x_{i}+1}^{i} \leq 2^{-1} \operatorname{diam} K_{x_{i}}^{i-1} .
$$

Since $K_{x_{i}}^{i-1} \backslash \bigcup_{j=i}^{\infty}\left(K_{x_{j}}^{j-1} \backslash K_{x_{j}+1}^{j}\right)$ consists of at most one point, we get

$$
\limsup _{i \rightarrow \infty} \operatorname{dim}_{H}\left(K_{x_{i}}^{i-1} \backslash K_{x_{i+1}}^{i}\right)=\infty .
$$


By taking a subsequence we may assume that $\lim _{i \rightarrow \infty} \operatorname{dim}_{H}\left(K_{x_{i}}^{i-1} \backslash K_{x_{i+1}}^{i}\right)=\infty$. Taking a closed $K_{i} \subseteq K_{x_{i}}^{i-1} \backslash K_{x_{i+1}}^{i}$ such that $\operatorname{dim}_{H} K_{i} \geq 2^{-1} \operatorname{dim}_{H}\left(K_{x_{i}}^{i-1} \backslash K_{x_{i+1}}^{i}\right)$ we easily see that this $\left\{K_{i}\right\}_{i=1}^{\infty}$ is the desired sequence.

We first prove Theorem 1.4. It turns out that Theorem 1.2 follows from the proof of Theorem 1.4.

Proof of Theorem 1.4. We take a sequence $\left\{K_{i}\right\}_{i=1}^{\infty}$ of closed subsets of $X$ in Lemma 2.1. For each $i$ we fix an element $x_{i} \in K_{i}$. Note that closed subsets of analytic sets are also analytic sets. By Theorem 1.3 there exist $A_{i} \subseteq K_{i}$ such that $\lim _{i \rightarrow \infty} \operatorname{dim}_{H} A_{i}=\infty$ and $A_{i}$ embeds into some ultrametric space $\left(U_{i}, \rho_{i}\right)$ with distortion $2+\varepsilon$, i.e., there exist $f_{i}: A_{i} \rightarrow U_{i}$ satisfying

$$
d(x, y) \leq \rho_{i}\left(f_{i}(x), f_{i}(y)\right) \leq(2+\varepsilon) d(x, y) \text { for any } x, y \in A_{i} .
$$

We divide the proof into three cases.

Case 1. $\left\{x_{i}\right\}_{i=1}^{\infty}$ is not bounded.

By taking a subsequence we may assume that $\lim _{n \rightarrow \infty} d\left(x_{1}, x_{i}\right)=\infty$ and that diam $K_{i} \leq 1 /(2+\varepsilon)$. By taking a further subsequence we may also assume that

$$
1 \leq \min \left\{\frac{\sqrt{1+\varepsilon}-1}{\sqrt{1+\varepsilon} \sqrt{1+2^{-1} \varepsilon}}, \frac{\sqrt{1+\varepsilon}-\sqrt{1+2^{-1} \varepsilon}}{\sqrt{1+2^{-1} \varepsilon}}, \frac{\sqrt{1+2^{-1} \varepsilon}-1}{2}\right\} d\left(A_{1}, A_{2}\right)
$$

and

$$
d\left(A_{1}, A_{i-1}\right) \leq \min \left\{\frac{\sqrt{1+\varepsilon}-1}{\sqrt{1+\varepsilon} \sqrt{1+2^{-1} \varepsilon}}, \frac{\sqrt{1+\varepsilon}-\sqrt{1+2^{-1} \varepsilon}}{\sqrt{1+2^{-1} \varepsilon}}\right\} d\left(A_{1}, A_{i}\right)
$$

for any $i \geq 2$. Put $R_{i}:=d\left(A_{i}, A_{1}\right)$ for $i \geq 2$. Note that diam $f_{i}\left(A_{i}\right) \leq 1$ since $f_{i}$ satisfies (2-1) and diam $A_{i} \leq \operatorname{diam} K_{i} \leq 1 /(2+\varepsilon)$.

For each $i \geq 2$ we take a point $u_{i, 0}$ not in $f_{i}\left(A_{i}\right)$ and put $Y_{i}:=f_{i}\left(A_{i}\right) \cup\left\{u_{i, 0}\right\}$. Define the distance function $\tilde{\rho}_{i}$ on $Y_{i}$ as follows: $\tilde{\rho}_{i}\left(u, u_{i, 0}\right):=R_{i}$ for $u \in f_{i}\left(A_{i}\right)$ and $\tilde{\rho}_{i}(u, v):=\rho_{i}(u, v)$ for $u, v \in f_{i}\left(A_{i}\right)$. Since diam $f_{i}\left(A_{i}\right) \leq 1 \leq R_{i}$, each $\left(Y_{i}, \tilde{\rho}_{i}\right)$ is an ultrametric space. Let us consider the space

$$
U:=\left\{\left(u_{i}\right) \in \prod_{i=2}^{\infty} Y_{i} \mid u_{i} \neq u_{i, 0} \text { only for finitely many } i\right\}
$$

and define the distance function $\rho$ on $U$ by

$$
\rho\left(\left(u_{i}\right),\left(v_{i}\right)\right):=\sup _{i} \tilde{\rho}_{i}\left(u_{i}, v_{i}\right) .
$$

It is easy to verify that $(U, \rho)$ is an ultrametric space. For each $x \in A_{i}$ we put

$$
f(x):=\left(u_{2,0}, u_{3,0}, \ldots, u_{i-1,0}, f_{i}(x), u_{i+1,0}, u_{i+2,0}, \ldots\right) .
$$


We shall prove that $f$ is a $(2+\varepsilon)$-embedding from the closed subset $\bigcup_{i=2}^{\infty} A_{i} \subseteq X$ to the ultrametric space $(U, \rho)$. Note that $\operatorname{dim}_{H}\left(\bigcup_{i=2}^{\infty} A_{i}\right)=\infty$.

We take two arbitrary points $x \in A_{i}$ and $y \in A_{j}(i<j)$ and fix $z \in A_{1}$. By (2-2) and (2-3), we get

$$
d(x, z) \leq R_{i}+\operatorname{diam} A_{1}+\operatorname{diam} A_{i} \leq R_{i}+2 \leq \sqrt{1+2^{-1} \varepsilon} R_{i} .
$$

Combining this inequality with (2-2) and (2-3) also implies

$$
\begin{aligned}
d(x, y) \geq d(y, z)-d(x, z) & \geq R_{j}-\sqrt{1+2^{-1} \varepsilon} R_{i} \\
& \geq \frac{1}{\sqrt{1+\varepsilon}} R_{j}=\frac{1}{\sqrt{1+\varepsilon}} \rho(f(x), f(y)),
\end{aligned}
$$

and

$$
\begin{aligned}
d(x, y) \leq d(x, z)+d(y, z) & \leq \sqrt{1+2^{-1} \varepsilon} R_{i}+\sqrt{1+2^{-1} \varepsilon} R_{j} \\
& \leq \sqrt{1+\varepsilon} R_{j}=\sqrt{1+\varepsilon} \rho(f(x), f(y)) .
\end{aligned}
$$

Hence $f$ is a $(2+\varepsilon)$-embedding.

Case 2. $\left\{x_{i}\right\}_{i=1}^{\infty}$ is bounded but not totally bounded.

By taking a subsequence, we may assume that there exist two constants $c_{1}, c_{2}>0$ such that

$$
c_{1} \leq d\left(x_{i}, x_{j}\right) \leq c_{2} \text { for any distinct } i, j .
$$

For any $\delta>0$ we divide $\left[c_{1}, c_{2}\right]=\bigcup_{j=1}^{m} I_{j}$ so that diam $I_{j}<\delta$ for any $j$.

Pick $j_{1} \in\{1,2, \ldots, m\}$ such that $d\left(x_{i}, x_{1}\right) \in I_{j_{1}}$ holds for infinitely many $i$. Put

$$
X_{1}:=\left\{x_{i} \mid d\left(x_{i}, x_{1}\right) \in I_{j_{1}}\right\}=\left\{x_{k_{1}(1)}, x_{k_{1}(2)}, \ldots\right\} .
$$

We then choose $j_{2} \in\{1,2, \ldots, m\}$ so that $d\left(x_{k_{1}(i)}, x_{k_{1}(1)}\right) \in I_{j_{2}}$ holds for infinitely many $i$ and put

$$
X_{2}:=\left\{x_{k_{1}(i)} \in X_{1} \mid d\left(x_{k_{1}(i)}, x_{k_{1}(1)}\right) \in I_{j_{2}}\right\}=\left\{x_{k_{2}(1)}, x_{k_{2}(2)}, \ldots\right\} .
$$

Repeatedly we obtain a sequence $\left\{j_{i}\right\}_{i=1}^{\infty}$ whose terms are elements of the set $\{1,2, \ldots, m\}$ and $X_{i}=\left\{x_{k_{i}(1)}, x_{k_{i}(2)}, \ldots\right\}$. By a pigeonhole argument we find a subsequence $\left\{j_{h(i)}\right\}_{i=1}^{\infty} \subseteq\left\{j_{i}\right\}_{i=1}^{\infty}$ that is monochromatic, i.e., $j_{h(i)} \equiv l$ for some $l \in\{1,2, \ldots, m\}$. We then get $d\left(x_{k_{h(i)}(i)}, x_{k_{h(j)}(j)}\right) \in I_{l}$. Since diam $I_{l}<\delta$ and $\lim _{i \rightarrow \infty} \operatorname{diam} A_{i}=0$, by choosing sufficiently small $\delta$ and taking a subsequence, we see that there exists a number $\alpha \geq c_{1}$ such that

$$
\alpha \leq d(u, v) \leq(1+\varepsilon) \alpha \quad \text { for any } u \in A_{i} \text { and } v \in A_{j}(i \neq j)
$$

and diam $A_{i} \leq(2+\varepsilon)^{-1} \alpha$. As in Case 1 we take a point $u_{i, 0}$ not in $f_{i}\left(A_{i}\right)$ and put 
$Y_{i}:=f_{i}\left(A_{i}\right) \cup\left\{u_{i, 0}\right\}$. We define the distance function $\tilde{\rho}_{i}$ on $Y_{i}$ by

$$
\tilde{\rho}_{i}\left(u, u_{i, 0}\right):=\alpha \quad \text { and } \quad \tilde{\rho}_{i}(u, v):=\rho_{i}(u, v)
$$

for $u, v \in f_{i}\left(A_{i}\right)$. Since $\operatorname{diam} f_{i}\left(A_{i}\right) \leq(2+\varepsilon) \operatorname{diam} A_{i} \leq \alpha$, each $\left(Y_{i}, \tilde{\rho}_{i}\right)$ is an ultrametric space. From these $\left(Y_{i}, \tilde{\rho}_{i}\right)$ we construct an ultrametric space $(U, \rho)$ by (2-4) and (2-5). Then a map $f: \bigcup_{i=2}^{\infty} A_{i} \rightarrow(U, \rho)$ defined by $(2-6)$ is a $(2+\varepsilon)$-embedding.

Case 3. $\left\{x_{i}\right\}_{i=1}^{\infty}$ is totally bounded.

The proof is similar to Case 1. From total boundedness, by taking a subsequence, we may assume that $\left\{x_{i}\right\}_{i=1}^{\infty}$ is a Cauchy sequence. Since $\lim _{i \rightarrow \infty} \operatorname{diam} A_{i}=0$, the sequence $\left\{A_{i}\right\}_{i=1}^{\infty}$ Hausdorff converges to a point $x_{\infty}$. Let $\delta>0$ be specified later. Note that $x_{\infty} \notin A_{i}$ for any sufficiently large $i$ since $A_{i}$ are mutually disjoint closed subsets of $X$. Hence, by taking a subsequence, we may also assume that $d\left(A_{i}, x_{\infty}\right) / d\left(A_{i-1}, x_{\infty}\right) \leq \delta$ for each $i$. Covering $A_{i}$ by countably many closed subsets $\left\{B_{i j}\right\}_{j}$ of diameter $\leq \delta d\left(A_{i}, x_{\infty}\right)$ we find a subset $B_{i j}$ such that $\operatorname{dim}_{H}\left(B_{i j}\right) \geq$ $2^{-1} \operatorname{dim}_{H}\left(A_{i}\right)$ and

$$
\frac{\operatorname{diam} B_{i j}}{d\left(B_{i j}, x_{\infty}\right)} \leq \frac{\operatorname{diam} B_{i j}}{d\left(A_{i}, x_{\infty}\right)} \leq \delta
$$

Hence by replacing $A_{i}$ with $B_{i j}$, we may assume that diam $A_{i} / d\left(A_{i}, x_{\infty}\right) \leq \delta$ for every $i$.

As in Cases 1 and 2 we add a point $u_{i, 0}$ to $f_{i}\left(A_{i}\right)$ and put $Y_{i}:=f_{i}\left(A_{i}\right) \cup\left\{u_{i, 0}\right\}$. Define the distance function $\tilde{\rho}_{i}$ on $Y_{i}$ by

$$
\tilde{\rho}_{i}\left(u, u_{i, 0}\right):=d\left(A_{i}, x_{\infty}\right) \quad \text { and } \quad \tilde{\rho}_{i}(u, v):=\rho_{i}(u, v)
$$

for $u, v \in f_{i}\left(A_{i}\right)$. If $\delta \leq(2+\varepsilon)^{-1}$, then we have

$$
\operatorname{diam} f_{i}\left(A_{i}\right) \leq(2+\varepsilon) \operatorname{diam} A_{i} \leq d\left(A_{i}, x_{\infty}\right),
$$

which implies that each $\left(Y_{i}, \tilde{\rho}_{i}\right)$ is an ultrametric space. From these $\left(Y_{i}, \tilde{\rho}_{i}\right)$ we define an ultrametric space $(U, \rho)$ by (2-4) and (2-5). If we trace the proof of Case 1 by replacing $R_{i}$ with $d\left(A_{i}, x_{\infty}\right)$, then we easily see that a map $f: \bigcup_{i=2}^{\infty} A_{i} \rightarrow(U, \rho)$ defined by $(2-6)$ is a $(2+\varepsilon)$-embedding, provided that $\delta>0$ is small enough.

Proof of Theorem 1.2. Let $X:=\left\{x_{1}, x_{2}, \ldots\right\}$. Apply the proof of Theorem 1.4 by identifying each $x_{i}$ with $K_{i}$. Note that the loss of the distortion in the proof only comes from (2-1), which we can ignore in the case where $A_{i}=x_{i}$. Hence the space $X$ can be embedded into an ultrametric space with distortion $1+\varepsilon$.

Remark 2.2. After this work was completed, the author proved in [Funano 2012] that every proper ultrametric space isometrically embeds into $\ell_{p}$ for any $p \geq 1$. In particular the subset $S$ in Theorem 1.3 also embeds into $\ell_{p}$. Theorems 1.2 and 1.4 
also hold in the case where the target metric space is $\ell_{p}$ instead of an ultrametric space. In fact, in the proof of Theorem 1.4, observe that we may assume that $A_{i}$ is compact [Carleson 1967; Howroyd 1995, Corollary 7]. Since $\bigcup_{i=2}^{\infty} A_{i}$ is a proper subset which embeds into an ultrametric space in the case of Cases 1 and 3, we consider only Case 2. Since we have (2-7) in Case 2 we easily see that $\bigcup_{i=2}^{\infty} A_{i}$ embeds into $\ell_{p}$. It was mentioned in [Funano 2012, Proposition 3.4] that an $\ell_{p}$ analogue of Theorem 1.5 also holds.

\section{Acknowledgements}

The author would like to express his thanks to Mr. Takumi Yokota for his suggestion regarding the two theorems in this paper and Mr. Ryokichi Tanaka for discussion. The author also thanks Professor Manor Mendel and Professor Assaf Naor for their useful comments. The author is also indebted to an anonymous referee for carefully reading this paper and making helpful comments.

\section{References}

[Albiac and Kalton 2006] F. Albiac and N. J. Kalton, Topics in Banach space theory, Graduate Texts in Mathematics 233, Springer, New York, 2006. MR 2006h:46005 Zbl 1094.46002

[Bartal et al. 2005] Y. Bartal, N. Linial, M. Mendel, and A. Naor, "On metric Ramsey-type phenomena”, Ann. of Math. (2) 162:2 (2005), 643-709. MR 2006g:46035 Zbl 1114.46007

[Bourgain et al. 1986] J. Bourgain, T. Figiel, and V. D. Milman, "On Hilbertian subsets of finite metric spaces”, Israel J. Math. 55:2 (1986), 147-152. MR 87m:46051 Zbl 0634.46008

[Carleson 1967] L. Carleson, Selected problems on exceptional sets, Van Nostrand Mathematical Studies 13, Van Nostrand, Princeton, NJ, 1967. MR 37 \#1576 Zbl 0189.10903

[Dvoretzky 1961] A. Dvoretzky, "Some results on convex bodies and Banach spaces", pp. 123-160 in Proceedings of the International Symposium on Linear Spaces (Jerusalem, 1960), Jerusalem Academic Press, 1961. MR 25 \#2518 Zbl 0119.31803

[Funano 2012] K. Funano, "Embedding proper ultrametric spaces into $\ell_{p}$ and its application to nonlinear Dvoretzky's theorem”, preprint, 2012. arXiv 1203.1761

[Grothendieck 1953] A. Grothendieck, "Sur certaines classes de suites dans les espaces de Banach et le théorème de Dvoretzky-Rogers", Bol. Soc. Mat. São Paulo 8 (1953), 81-110. MR 20 \#1195

[Howroyd 1995] J. D. Howroyd, "On dimension and on the existence of sets of finite positive Hausdorff measure", Proc. London Math. Soc. (3) 70 (1995), 581-604. MR 96b:28004 Zbl 0828.28002

[Kechris 1995] A. S. Kechris, Classical descriptive set theory, Graduate Texts in Mathematics 156, Springer, New York, 1995. MR 96e:03057 Zbl 0819.04002

[Mendel and Naor 2007] M. Mendel and A. Naor, "Ramsey partitions and proximity data structures", J. Eur. Math. Soc. (JEMS) 9:2 (2007), 253-275. MR 2008i:46014 Zbl 1122.68043

[Mendel and Naor 2012] M. Mendel and A. Naor, "Ultrametric subsets with large Hausdorff dimension", Invent. Math. (2012). arXiv 1106.0879v3

[Milman 1971] V. D. Milman, "A new proof of A. Dvoretzky's theorem on cross-sections of convex bodies", Funkcional. Anal. i Priložen. 5:4 (1971), 28-37. In Russian; translated in Funct. Anal. Appl. 5:4 (1971), 288-295. MR 45 \#2451 Zbl 0239.46018 
[Milman 1992] V. D. Milman, “Dvoretzky's theorem: thirty years later”, Geom. Funct. Anal. 2:4 (1992), 455-479. MR 93i:46002 Zbl 0787.46016

[Milman and Schechtman 1999] V. D. Milman and G. Schechtman, "An "isomorphic" version of Dvoretzky's theorem, II”, pp. 159-164 in Convex geometric analysis (Berkeley, CA, 1996), edited by K. M. Ball and V. D. Milman, Math. Sci. Res. Inst. Publ. 34, Cambridge University Press, Cambridge, 1999. MR 2000c:46016 Zbl 0942.46012

[Naor and Tao 2012] A. Naor and T. Tao, "Scale-oblivious metric fragmentation and the nonlinear Dvoretzky theorem”, Israel J. Math. (2012).

[Schechtman 2006] G. Schechtman, "Two observations regarding embedding subsets of Euclidean spaces in normed spaces", Adv. Math. 200:1 (2006), 125-135. MR 2006j:46015 Zbl 1108.46011

[Schechtman 2011] G. Schechtman, "Euclidean sections of convex bodies, series of lectures", preprint, 2011. arXiv 1110.6401

[Vestfrid and Timan 1979] I. A. Vestfrid and A. F. Timan, "A universal property of Hilbert spaces", Dokl. Akad. Nauk SSSR 246:3 (1979), 528-530. In Russian; translated in Sov. Math. Dokl. 20 (1979), 485-486. MR 80e:54039 Zbl 0423.54007

Received November 6, 2011. Revised April 16, 2012.

KEI FUNANO

RESEARCh Institute FOR Mathematical SCIENCES

KYOTO UNIVERSITY

КҮОТО 606-8502

JAPAN

kfunano@kurims.kyoto-u.ac.jp

https://sites.google.com/site/keifunanoshomepage/ 


\title{
PACIFIC JOURNAL OF MATHEMATICS
}

\author{
http://pacificmath.org \\ Founded in 1951 by \\ E. F. Beckenbach (1906-1982) and F. Wolf (1904-1989)
}

\section{EDITORS}

V. S. Varadarajan (Managing Editor)

Department of Mathematics

University of California

Los Angeles, CA 90095-1555

pacific@math.ucla.edu

Vyjayanthi Chari

Department of Mathematics

University of California

Riverside, CA 92521-0135

chari@math.ucr.edu

\section{Robert Finn}

Department of Mathematics Stanford University

Stanford, CA 94305-2125

finn@math.stanford.edu

Kefeng Liu

Department of Mathematics

University of California

Los Angeles, CA 90095-1555

liu@math.ucla.edu
Darren Long

Department of Mathematics

University of California

Santa Barbara, CA 93106-3080

long@math.ucsb.edu

Jiang-Hua Lu

Department of Mathematics

The University of Hong Kong

Pokfulam Rd., Hong Kong jhlu@maths.hku.hk

Alexander Merkurjev

Department of Mathematics

University of California

Los Angeles, CA 90095-1555

merkurev@math.ucla.edu
Sorin Popa

Department of Mathematics University of California

Los Angeles, CA 90095-1555 popa@math.ucla.edu

Jie Qing

Department of Mathematics

University of California

Santa Cruz, CA 95064

qing@cats.ucsc.edu

Jonathan Rogawski

Department of Mathematics

University of California

Los Angeles, CA 90095-1555

jonr@math.ucla.edu

\section{PRODUCTION}

pacific@math.berkeley.edu

\section{SUPPORTING INSTITUTIONS}

ACADEMIA SINICA, TAIPEI

CALIFORNIA INST. OF TECHNOLOGY INST. DE MATEMÁTICA PURA E APLICADA KEIO UNIVERSITY

MATH. SCIENCES RESEARCH INSTITUTE NEW MEXICO STATE UNIV.

OREGON STATE UNIV.

\author{
STANFORD UNIVERSITY \\ UNIV. OF BRITISH COLUMBIA \\ UNIV. OF CALIFORNIA, BERKELEY \\ UNIV. OF CALIFORNIA, DAVIS \\ UNIV. OF CALIFORNIA, LOS ANGELES \\ UNIV. OF CALIFORNIA, RIVERSIDE \\ UNIV. OF CALIFORNIA, SAN DIEGO \\ UNIV. OF CALIF., SANTA BARBARA
}

\author{
UNIV. OF CALIF., SANTA CRUZ \\ UNIV. OF MONTANA \\ UNIV. OF OREGON \\ UNIV. OF SOUTHERN CALIFORNIA \\ UNIV. OF UTAH \\ UNIV. OF WASHINGTON \\ WASHINGTON STATE UNIVERSITY
}

These supporting institutions contribute to the cost of publication of this Journal, but they are not owners or publishers and have no responsibility for its contents or policies.

See inside back cover or pacificmath.org for submission instructions.

The subscription price for 2012 is US \$420/year for the electronic version, and \$485/year for print and electronic.

Subscriptions, requests for back issues from the last three years and changes of subscribers address should be sent to Pacific Journal of Mathematics, P.O. Box 4163, Berkeley, CA 94704-0163, U.S.A. Prior back issues are obtainable from Periodicals Service Company, 11 Main Street, Germantown, NY 12526-5635. The Pacific Journal of Mathematics is indexed by Mathematical Reviews, Zentralblatt MATH, PASCAL CNRS Index, Referativnyi Zhurnal, Current Mathematical Publications and the Science Citation Index.

The Pacific Journal of Mathematics (ISSN 0030-8730) at the University of California, c/o Department of Mathematics, 969 Evans Hall, Berkeley, CA 94720-3840, is published monthly except July and August. Periodical rate postage paid at Berkeley, CA 94704, and additional mailing offices. POSTMASTER: send address changes to Pacific Journal of Mathematics, P.O. Box 4163, Berkeley, CA 94704-0163.

PJM peer review and production are managed by EditFLOW ${ }^{\mathrm{TM}}$ from Mathematical Sciences Publishers.

PUBLISHED BY PACIFIC JOURNAL OF MATHEMATICS

at the University of California, Berkeley 94720-3840

A NON-PROFIT CORPORATION

Typeset in LATEX

Copyright $(02012$ by Pacific Journal of Mathematics 


\title{
PACIFIC JOURNAL OF MATHEMATICS
}

\author{
Volume $259 \quad$ No. $1 \quad$ September 2012
}

Extension Theorems for external cusps with minimal regularity

GABRIEL ACOSTA and IGNACIO OJEA

Convergence of axially symmetric volume-preserving mean curvature flow

MARIA ATHANASSENAS and SEVVANDi KANDANAARACHCHI

On the horoboundary and the geometry of rays of negatively curved manifolds

FranÇOISE DAL'bo, Marc PEIGNÉ and ANDREA

SAMBUSETTI

Two infinite versions of the nonlinear Dvoretzky theorem

KEI FUNANO

Nonlocal uniform algebras on three-manifolds

ALEXANDER J. IZZO

Mahlo cardinals and the torsion product of primary abelian groups

PATRICK W. KEEF

Geometry of trinomials

AARON MELMAN

Drinfeld orbifold algebras

ANNE V. SHEPLER and SARAH Witherspoon

Semi-topological cycle theory I

JYH-HAUR TEH

New construction of fundamental domains for certain Mostow groups 209

TiEHONG ZHAO 